\title{
Stability of functionally graded shape memory alloy sandwich panels
}

\author{
Victor Birman \\ University of Missouri-Rolla, Engineering Education Center, \\ 8001 Natural Bridge Road, St Louis, MO 63121, USA
}

Received 18 August 1995, accepted for publication 31 January 1997

\begin{abstract}
The stability of sandwich panels subjected to the simultaneous action of a uniform temperature and a uniaxial compression is considered. At elevated temperatures, the buckling load can be increased by using shape memory alloy (SMA) fibers in resin sleeves embedded within the core, at the midplane of the sandwich panel. The best results are achieved when the spacing of SMA fibers across the panel is nonuniform, i.e. the spacing, which is minimum at the centerline, gradually increases with the approach to the edges (sinusoidal distribution). The effectiveness of SMA fibers increases with temperature due to larger tensile recovery stresses. The example of stability of sandwich panels considered in the paper illustrates that functionally graded SMA composites may present significant advantages in engineering design.
\end{abstract}

\section{Introduction}

Shape memory alloys (SMAs) have recently been investigated as a possible tool for modification of the structural response. An attempt was undertaken to incorporate SMAs within a composite material in the form of 'smart fibers'. The feasibility of such hybrid materials has been recently illustrated by White et al (1993) who used two-way shape memory training.

There are relatively few analytical studies of SMA composites. Among these, Rogers et al (1991) employed a rule of mixtures to characterize composite plates consisting of graphite/epoxy and Nitinol/epoxy layers. The same rule was used by Pates et al (1994) and Zhong et al (1994) who considered thermal buckling and random vibrations of SMA composite plates with Nitinol fibers embedded within composite layers. A representation of the constitutive behavior of SMA composites based on advanced micromechanical theories has also attracted significant interest. In particular, Boyd and Lagoudas (1993) developed constitutive relations for SMA composites based on Mori-Tanaka micromechanics. A theory based on the multi-cell micromechanics of Chamis has recently been proposed by Saravanos et al (1996).

A different approach based on embedding SMA fibers in vulcanized rubber sleeves within the composite material was proposed by Baz and Ro (1992) and Baz and Chen (1993). This method simplifies the manufacturing process and eliminates the problem of decay of the shape memory effect in systems that include SMA fibers bonded to a composite material. Experimental verification of the theoretical model presented by Baz and Chen (1993) illustrated promising features of the method. The same approach has recently been employed by Epps and Chandra (1995) whose experimental results were found to be in good agreement with theoretical predictions.

There are two methods to describe the behavior of a SMA material. Constitutive theories were developed by Baumgart et al (1976), Tanaka (1986), Sato and Tanaka (1988), Liang and Rogers (1990), Brinson (1993) and others. Comprehensive reviews of these theories can be found in works by Jia et al (1996) and Birman (1997). A different approach is based on experimental constitutive curves that were published for particular materials subjected to a prescribed loading regime. While this approach lacks the universal features of an analytical method, it provides a convenient tool to prescribe the response of a particular material. In particular, Cross et al (1970) published constitutive relationships for 55-Nitinol, an alloy that contains between 52 and 56 weight per cent of nickel, the rest being titanium. Tensile recovery stress-temperature curves for $100 \mathrm{~mm}$ diameter rods presented in this report represent an example of the experimental data necessary for the design of shape memory fibers. Baz and Ro (1992) and Epps and Chandra (1995) also presented constitutive curves for Nitinol.

The concept of functionally graded composite materials has become an important area of research (see, for example, special issues of Composites Engineering in 1994 (vol 4, no 1) and 1995 (vol 5, no 7)). These materials are characterized by concentrations of the fibers in the most critical regions where higher fiber volume fractions can enhance the response. A further step is represented by functionally graded hybrid composite materials including more than one class of fiber. In particular, functionally graded materials consisting of two different classes of 
fibers and their application in the stability problem were discussed by Birman (1995). The present paper addresses a different problem, i.e. smart (SMA) fibers embedded within the core of a sandwich panel and nonuniformly distributed to achieve maximum stability. The class of structures considered here represents functionally graded smart composite structures.

It should be noted that the design of functionally graded structures is closely related to the problem of optimization. Studies dealing with the optimization of smart (usually piezoelectric) composite structures have been published (Clark and Fuller 1992, Birman and Adali 1996, Birman and Simonyan 1996), however the concept of functionally graded smart composite structures advocated in this paper has not yet been considered, to the best knowledge of the author.

In the present paper, SMA fibers in sleeves are embedded within a sandwich panel to enhance its stability when subjected to the simultaneous action of a uniform thermal field and a compressive load. The presence of a uniform temperature is due to the external environment (e.g. aerodynamic heating of the structure). This simplifies the solution compared to the case where SMA fibers are activated by an electric current, in which case the temperature is nonuniformly distributed over the cross section. An interesting feature of structures where the reverse phase transformation of SMA fibers is triggered by an external temperature is that an active control is actually replaced by a passive control of the structure. This means that there is no need for phase transformation in external power sources.

\section{Analysis}

Consider a rectangular sandwich panel subjected to the simultaneous action of an elevated temperature $T$ and compressive forces of intensity $N_{x}$, as shown in figure 1 . SMA fibers in resin sleeves are embedded within the core, in the middle plane of the sandwich. The fibers are originally in the austenitic phase. Then the temperature is reduced so that the fiber material is transformed into the martensitic phase. The martensitic fibers are prestrained and their ends are clamped. Now, if the temperature is increased, the material experiences the reverse transformation into austenite accompanied by a contraction to the original length. However, this contraction is prevented by clamping and as a result, tensile recovery stresses appear in the fibers. The fibers are oriented in the load direction so that tensile recovery forces enhance stability. In general, these forces can be generated as a result of heating due to an electric current in SMA fibers. On the negative side, this would result in additional thermal compressive forces as well as degradation of the properties of the core and composite facings. However, in numerous applications, a structure operates under an elevated temperature that is sufficient to cause the reverse transformation of martensitic SMA fibers. Such is the case in aerospace structures where elevated temperatures result from aerodynamic heating or from the heat generated by the engines. In these situations, activation of SMA components
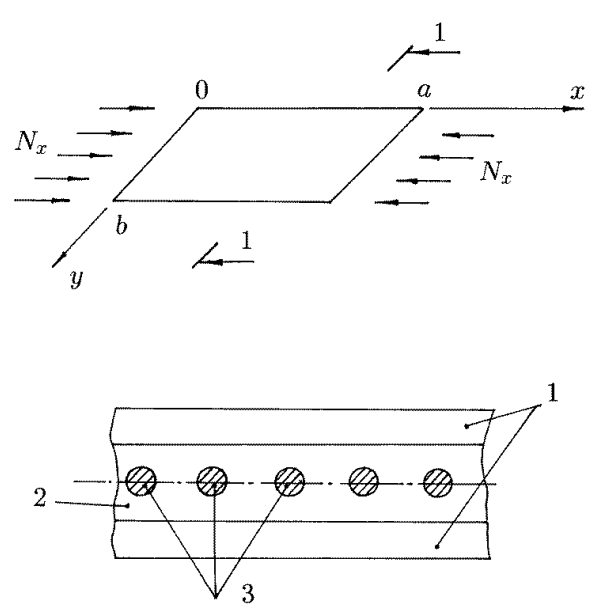

Figure 1. Sandwich panel and its cross section with SMA fibers in sleeves: 1, facings; 2 , core; 3, SMA fibers in sleeves.

may occur simultaneously with loading, i.e. the structure experiences a passive control instead of an active control.

A potential drawback in the design discussed here is related to the necessity to isolate the forces applied by SMA fibers to the clamps from the supported structure. Otherwise, these forces will cancel the beneficial effect of the method. The solution may be to support several spans of the panel using continuous SMA fibers. The intermediate supports between adjacent spans can be designed to prevent transverse deformations, without restricting axial displacements of SMA fibers. Therefore, only the outer supports will be loaded by the forces from SMA wire. It is important to emphasize that these forces are independent of the length of the fibers, i.e. the proposed arrangement can be used to enhance the stability of long structures.

The sandwich is characterized using the theory presented by Whitney (1987) that employs the following major assumptions:

(1) the facings are in the state of plane stress;

(2) the core can translate transverse shearing stresses only;

(3) perfect bonding exists between all elements of the sandwich.

Under these assumptions, the behavior of the panel can be described using the first-order shear deformation theory. The panels considered in this paper are symmetric with respect to the middle plane. Each facing is composed from multiple angle-ply and specially orthotropic layers so that the bending stiffnesses $D_{16}$ and $D_{26}$ are negligible compared to other bending stiffnesses. This results in uncoupled in-plane and transverse static equilibrium equations.

In the present study, in-plane forces that affect the equations of equilibrium in the thickness direction include external loads $N_{x}$, thermal stress resultants $N_{x}^{T}, N_{y}^{T}$, and SMA recovery forces of intensity $N_{x}^{r}$. Accordingly, equations of equilibrium of the panel become:

$$
\begin{gathered}
A_{55}\left(w_{, x x}+\Psi_{x, x}\right)+A_{44}\left(w_{, y y}+\Psi_{y, y}\right)-N_{x}^{r} \Psi_{x, x} \\
+\left(N_{x}+N_{x}^{T}\right) \Psi_{x, x}+N_{y}^{T} \Psi_{y, y}=0
\end{gathered}
$$




$$
\begin{aligned}
& -A_{44}\left(w_{, y}+\Psi_{y}\right)+\left(D_{12}+D_{66}\right) \Psi_{x, x y}+D_{66} \Psi_{y, x x} \\
& \quad+D_{22} \Psi_{y, y y}=0 \\
& -A_{55}\left(w_{, x}+\Psi_{x}\right)+D_{11} \Psi_{x, x x}+D_{66} \Psi_{x, y y} \\
& \quad+\left(D_{12}+D_{66}\right) \Psi_{y, x y}=0
\end{aligned}
$$

where $w, \Psi_{x}$ and $\Psi_{y}$ are transverse deflection and bending slopes in the planes $x z$ and $y z$ respectively, and $A_{i i}, D_{i j}$ denote extensional and bending stiffnesses.

In the panels considered here bending stiffnesses are not significantly affected by SMA fibers. However, extensional stiffnesses $A_{44}$ and $A_{55}$ are reduced due to the loss of a part of the core cross sectional areas occupied by the fibers in sleeves. As follows from the formula for the modulus of an equivalent solid core derived in appendix A, this effect may be negligible in numerous applications. In particular, it can be disregarded for the core considered in the numerical examples presented in this paper. Therefore, in the following solution, the stiffnesses $A_{44}$ and $A_{55}$ are assumed constant and independent of the fibers in sleeves.

The recovery stress resultant can be calculated as

$$
N_{x}^{r}=\sigma_{r} \frac{A_{f}}{l}
$$

where $\sigma_{r}$ are recovery stresses, $A_{f}$ is the cross sectional area of a SMA fiber, and $l$ is the spacing of SMA fibers that may be a function of the $y$-coordinate. In the present paper, experimental results (Cross et al 1970) are used to characterize the recovery stress in Nitinol fibers as a function of temperature and the initial prestrain. This eliminates the necessity to employ analytical constitutive relationships that often overestimate the stresses in the regime of a constrained recovery.

Thermal stress resultants in the first equation (1) are the following functions of temperature:

$$
\left\{\begin{array}{c}
N_{x}^{T} \\
N_{y}^{T}
\end{array}\right\}=2 \int_{h_{f}}\left[\begin{array}{c}
Q_{11} Q_{12} \\
Q_{12} Q_{22}
\end{array}\right]\left\{\begin{array}{c}
\alpha_{x} \\
\alpha_{y}
\end{array}\right\}_{k}\left(T-T_{0}\right) \mathrm{d} z
$$

where $Q_{i j}$ are the transformed reduced stiffnesses of the $k$ th layer of the facings, $\alpha_{i}$ are the coefficients of thermal expansion of this layer, and $T_{0}$ is a reference temperature corresponding to zero thermal stress resultants. The terms proportional to $Q_{16}, Q_{26}$, and $\alpha_{x y}$ are negligible, similar to the stiffnesses $D_{16}$ and $D_{26}$, because the facings have many layers and they are symmetrically laminated with respect to the middle plane of the sandwich. The integration in equations (3) is carried out through the thickness of one of the facings. Note that in-plane shear thermal stress resultants are negligible. The contribution of SMA fibers to $N_{x}^{T}$ does not have to be accounted for in the above equation because it is included into experimentally evaluated recovery stresses.

The purpose here is to illustrate that functional grading, i.e. a nonuniform distribution of SMA fibers, can result in a significant enhancement of stability. Due to obvious technological reasons, the cross sectional areas of the fibers should remain constant throughout the entire structure. However, the spacing can be variable, i.e. $l=l(y)$. In this case, the recovery stress resultants $N_{x}^{r}$ depend on the $y$-coordinate. In the absence of SMA fibers, or even if they are present but uniformly distributed over the cross section, the mode shape of instability of a simply supported panel can be represented as:

$$
\begin{aligned}
w & =W_{m n} \sin \frac{m \pi x}{a} \sin \frac{n \pi y}{b} \\
\Psi_{x} & =H_{m n} \cos \frac{m \pi x}{a} \sin \frac{n \pi y}{b} \\
\Psi_{y} & =R_{m n} \sin \frac{m \pi x}{a} \cos \frac{n \pi y}{b}
\end{aligned}
$$

where $m$ and $n$ are integers. This mode shape satisfies boundary conditions for a panel supported by ribs (stiffeners) with an infinite bending stiffness $(w=0$ around the contour of the panel). The torsional stiffness of the ribs is negligible, but the shearing stiffness in the plane of the web is very high $\left(\Psi_{y}=0\right.$ along the edges $x=0$ and $x=a$, and $\Psi_{x}=0$ along $y=0$ and $y=b$ ). Obviously, such boundary conditions can adequately model designs where the panel is supported by a blade or other open profile stiffeners or by bulkheads. Although the buckling mode shape in the case of uniformly distributed external and recovery forces corresponds to $n=1$, other values of $n$ have to be examined in the present problem as well. This accounts for a possible situation where critical loads corresponding to the mode shape with $n=1$ become higher than their counterparts corresponding to larger values of $n$. Such situations may arise as a result of nonuniformly distributed recovery forces.

It seems reasonable to assume that maximum benefits to stability will be achieved if the distribution of tensile recovery forces generated by SMA fibers follows a sinusoidal law in the $y$-direction, i.e.

$$
N_{x}^{r}=\sigma_{r} A \sin \pi y / b
$$

where $A \sin \pi y / b=A_{f} / l(y)$. Obviously, the spacing corresponding to this distribution implies a large distance between SMA fibers in the vicinity of the edges $y=0$ and $y=b$. On the contrary, SMA fibers are located close to each other in the central section of the plate. The proof of the claim that equation (5) represents an optimum distribution of SMA fibers is given in appendix B for thin sandwich plates.

In reality, it is more likely that the spacing will change according to a piece-wise law that may approach a sinusoidal distribution, if the number of sections with a constant spacing of SMA fibers is sufficiently large.

The magnitude of $A$ is determined from the constraint on the total volume (cross sectional area) of SMA fibers:

$$
\int_{0}^{b} A \sin \frac{\pi y}{b} \mathrm{~d} y \leq A_{m}
$$

where $A_{m}$ is a constant. Obviously, $A=A_{m} \pi / 2 b$.

The substitution of equations (4) and (5) into equations (1) and the Galerkin procedure yield a set of homogeneous algebraic equations with respect to the amplitudes $W_{m n}, H_{m n}$ and $R_{m n}$. The requirement of a nonzero solution results in the buckling equation:

$$
\left|\begin{array}{ccc}
k_{11}^{(m n)} & k_{12}^{(m n)}+\frac{m \pi}{a}\left(N_{x}+N_{x}^{T}-\sigma_{r} A C^{(n)}\right)+\frac{n \pi}{b} N_{y}^{T} & k_{13}^{(m n)} \\
k_{12}^{(m n)} & k_{22}^{(m n)} & k_{23}^{(m n)} \\
k_{31}^{(m n)} & k_{32}^{(m n)} & k_{33}^{(m n)}
\end{array}\right|=0
$$


where the coefficients are given by

$$
\begin{gathered}
k_{11}^{(m n)}=\left(\frac{m \pi}{a}\right)^{2} A_{55}+\left(\frac{n \pi}{b}\right)^{2} A_{44} \\
k_{12}^{(m n)}=k_{21}^{(m n)}=\frac{m \pi}{a} A_{55} \\
k_{13}^{(m n)}=k_{31}^{(m n)}=\frac{n \pi}{b} A_{44} \\
k_{22}^{(m n)}=A_{55}+\left(\frac{m \pi}{a}\right)^{2} D_{11}+\left(\frac{n \pi}{b}\right)^{2} D_{66} \\
k_{23}^{(m n)}=k_{32}^{(m n)} \frac{m \pi}{a} \frac{n \pi}{b}\left(D_{12}+D_{66}\right) \\
k_{33}^{(m n)}=A_{44}+\left(\frac{n \pi}{b}\right)^{2} D_{22}+\left(\frac{m \pi}{a}\right)^{2} D_{66} .
\end{gathered}
$$

In equation (7), the coefficient $C^{(n)}$ reflects the effect of a nonuniform distribution of SMA fibers:

$$
C^{(n)}=\frac{1}{\pi} \frac{8 n^{2}}{4 n^{2}-1} .
$$

If SMA fibers are uniformly distributed, i.e. $l(y)=$ constant, $C(n)=1$. If the conclusion on the effectiveness of a nonuniform distribution of SMA fibers is based on the condition that the total volume of these fibers for both uniform and sinusoidal distributions must remain constant, in the case of a uniform distribution, the value of $A$ in equation (7) should be replaced with $(2 / \pi) A$.

To conclude this section, it is useful to estimate the potential for enhancement of the stability offered by optimization of the distribution of SMA fibers. If the effect of SMA fibers on the stiffnesses $A_{44}$ and $A_{55}$ can be disregarded, the analysis of the effectiveness of these fibers is reduced to a comparison of the term proportional to the recovery stress in equation (7). It can be immediately shown that a sinusoidal distribution of the recovery stress resultants given by equation (5) results in a gain of the effectiveness of SMA fibers by $33 \%$, as compared to a uniform distribution. This number is expected to be reduced if the stiffnesses are affected by a reduction of the cross sectional area associated with the sleeves of the embedded fibers. On the other hand, if technological difficulties of curing composites with embedded SMA fibers (without sleeves) can be overcome, the stiffnesses of the composite may actually increase due to the inclusion of SMA fibers. In this case, the benefits to stability will exceed $33 \%$.

\section{Numerical examples}

Two types of sandwich panels are considered in the numerical examples. The first type has isotropic aluminum facings (Al 6061-T6: $E=69.0 \mathrm{GPa}, G=26.0 \mathrm{GPa}$, $\left.\alpha=23.6 \times 10^{-6}{ }^{\circ} \mathrm{C}^{-1}\right)$. The facings of the second type are manufactured from Kevlar/epoxy with the following characteristics: $E_{1}=87.0 \mathrm{GPa}, E_{2}=5.5 \mathrm{GPa}, G_{12}=$ $2.2 \mathrm{GPa}, v_{12}=0.34, \alpha_{1}=-2.0 \times 10^{-6}{ }^{\circ} \mathrm{C}^{-1}, \alpha_{2}=$ $60.0 \times 10^{-6}{ }^{\circ} \mathrm{C}^{-1}$. The core material is Rohacell 51WP with a shear modulus equal to $20.6 \mathrm{MPa}$. This core material is selected for the analysis because it can withstand high temperatures of up to $177^{\circ} \mathrm{C}$. In all examples, the reference temperature is equal to $16^{\circ} \mathrm{C}$. The properties of materials considered in the examples are assumed independent of temperature.

The thickness of each facing is equal to $0.00254 \mathrm{~m}$. Kevlar/epoxy facings are composed of ten layers, each layer being $0.000254 \mathrm{~m}$ thick. The lamination of Kevlar/epoxy facings is: $\left[0^{\circ} / 45^{\circ} /-45^{\circ} / 45^{\circ} /-45^{\circ}\right]_{s}$. The core is $0.0127 \mathrm{~m}$ thick. In all examples, the in-plane panel dimensions were $a=1.0 \mathrm{~m}$ and $b=0.5 \mathrm{~m}$, if not indicated otherwise.

SMA fibers in sleeves are located at the middle plane of the sandwich. The diameter of the fibers is equal to $0.00254 \mathrm{~m}$, as was the case in the report of Cross et al (1970) whose experimental stress-temperature curve is used in the following examples. The external diameter of the sleeves is $0.00294 \mathrm{~m}$ corresponding to the thickness equal to $0.2 \mathrm{~mm}$ used by Baz and Chen (1993). The tensile recovery stress-temperature curve employed in the analysis corresponds to an initial strain equal to $8 \%$. As was shown by Cross et al (1970), such prestrain results in maximum recovery stresses at temperatures exceeding $120^{\circ} \mathrm{C}$.

The following results present a comparison of three cases, namely functionally graded SMA fibers corresponding to a sinusoidal distribution, uniformly distributed SMA fibers and panels without SMA fibers. The total volume of SMA fibers in the first two cases is kept equal.

The effect of SMA fibers on stability is illustrated in figures 2 and 3 for sandwich panels with aluminum and Kevlar/epoxy facings respectively. As follows from these figures, the influence of SMA fibers on stability is relatively weak at low temperatures. This is natural, because at these temperatures tensile recovery stresses in SMA fibers are low. The situation changes at higher temperatures due to two factors. First of all, significant thermal compressive stresses result in a dramatic reduction in the mechanical bucking load. Secondly, high recovery stresses in the SMA fibers enhance the stability of sandwich panels. Moreover, the advantage of the optimum distribution of SMA fibers becomes noticeable at higher temperatures and recovery stresses. For example, in the panels with aluminum facings, the improvement in the buckling load at $82{ }^{\circ} \mathrm{C}$ was $5.0 \%$ in the case of uniformly distributed SMA fibers and $6.6 \%$ in the case of a sinusoidal distribution. However, at $149^{\circ} \mathrm{C}$ these values increased to $75.5 \%$ and $100.8 \%$ respectively. In the panels with Kevlar/epoxy facings the buckling load at $82{ }^{\circ} \mathrm{C}$ was increased by $5.7 \%$ for uniformly distributed fibers and by $7.7 \%$ for functionally graded fibers. At $149^{\circ} \mathrm{C}$, the improvement was $26.9 \%$ and $35.8 \%$ respectively. The higher effectiveness of SMA fibers in the panels with aluminum facings at $149{ }^{\circ} \mathrm{C}$ is explained by the approach to the critical temperature that results in a sharp reduction of the mechanical buckling load.

Note that the instability mode shape in all examples considered in this paper corresponded to $n=1$. In the case of panels with aluminum facings (figure 2) the number of half-waves in the mode shape of buckling was equal to two $(m=2)$. However, for the panels with Kevlar/epoxy facings (figure 3), the mode shape at lower temperatures corresponded to three half-waves, while as the 


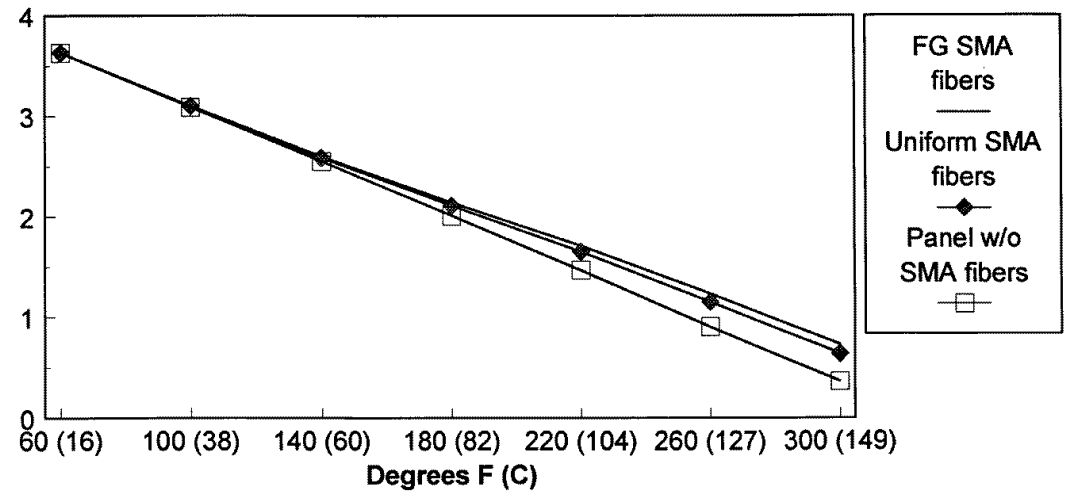

Figure 2. Buckling loads for panels with aluminum facings.

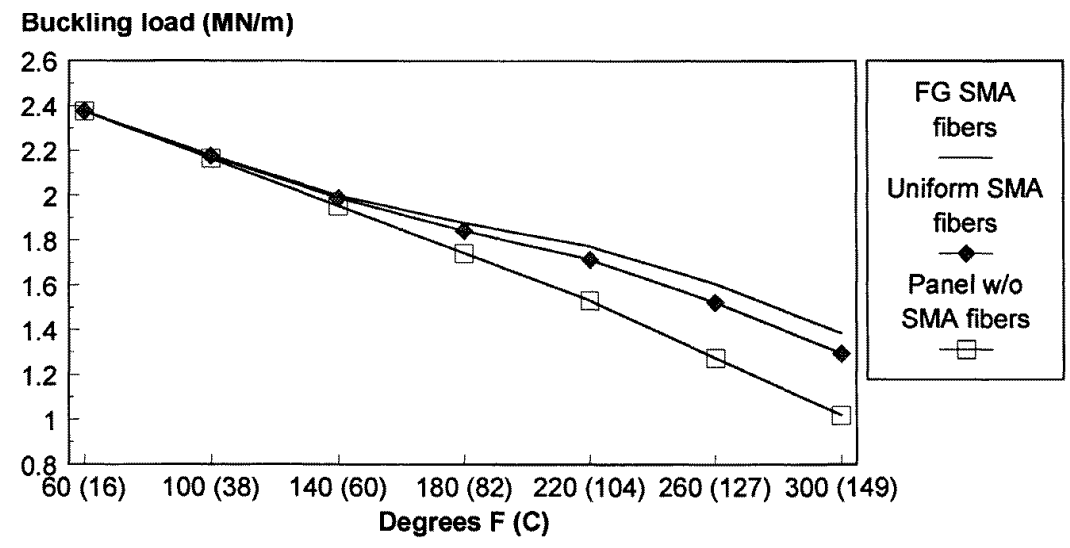

Figure 3. Buckling loads for panels with Kevlar/epoxy facings.

\section{Buckling load (MN/m)}

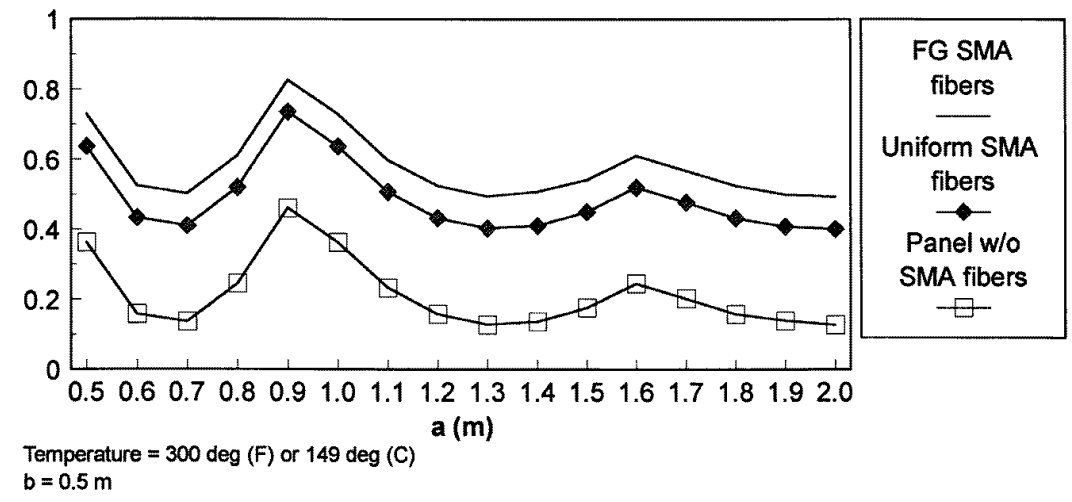

Figure 4. Effect of the aspect ratio on the buckling loads of panels with aluminum facings at $T=149^{\circ} \mathrm{C}$.

temperature increased beyond $120^{\circ} \mathrm{C}$, the buckling mode shape changed to $m=2$. Such a change in the buckling mode shape may be explained by the influence of biaxial thermal compressive forces.

The effect of SMA fibers on stability is illustrated for panels with various aspect ratios in figures 4 and 5 . Note that the change in buckling mode shape with aspect ratio is clearly evident in the case of aluminum facings (figure 4). In the case of Kevlar/epoxy facings this change is less pronounced. In the panels with aluminum facings, $m=1$ if $a<0.9, m=2$ if $0.9<a<1.7$, and $m=3$ if $1.7<a<2.0$. In the case of the panels with Kevlar/epoxy facings, $m=1$ if $a<0.6, m=2$ if $0.6<a<1.1, m=3$ if $1.1<a<1.5, m=4$ if $1.5<a<1.9$, and $m=5$ in the range $1.9<a<2.0$. Of course, all lengths ' $a$ ' listed above are measured in 
Buckling load (MN/m)

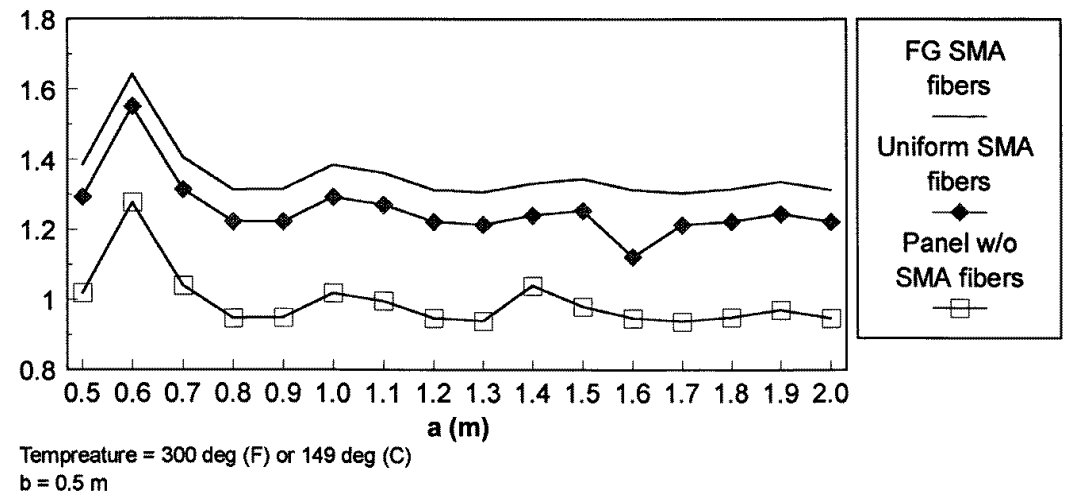

Figure 5. Effect of the aspect ratio on the buckling loads of panels with Kevlar/epoxy facings at $T=149^{\circ} \mathrm{C}$.

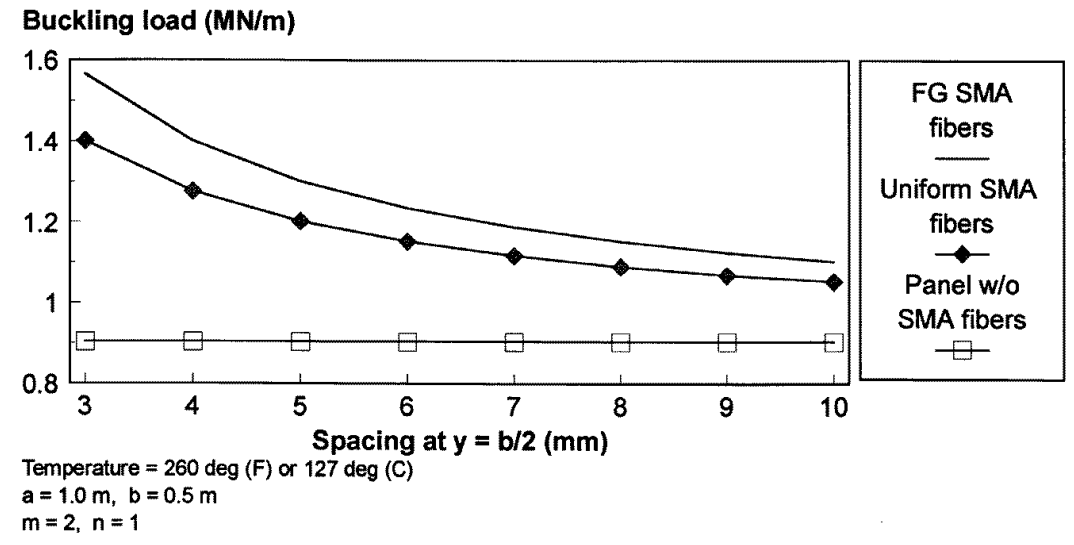

Figure 6. Effect of the spacing between SMA fibers on the buckling loads of panels with aluminium 127 facings at $T=127^{\circ} \mathrm{C}$.

metres. It is interesting to note that a consistent advantage of functionally graded SMA fibers over their uniformly distributed counterparts can be observed at all aspect ratios. In turn, the advantage in panels with uniformly distributed SMA fibers over those without such fibers is also unaffected by the aspect ratio.

Finally, the effect of the spacing of SMA fibers is illustrated for panels with aluminum facings (figure 6) and for their counterparts with Kevlar/epoxy facings (figures 7 and 8). The spacing shown along the horizontal axes of the figures corresponds to the minimum spacing of functionally graded fibers along the centerline $y=b / 2$. Note that, according to the previous discussion, the actual spacing of uniformly distributed fibers can be obtained from that shown in the figures by multiplication by the factor $\pi / 2$. This guarantees an equivalence of the volume of SMA fibers in both functionally graded and uniform configurations.

Predictably, in all cases the maximum effect was observed when the spacing was minimum. However, the accuracy of the results generated for a small spacing may be unsatisfactory. This is due to the fact that if the spacing is small it is impossible to neglect degradation of the core stiffnesses $A_{44}$ and $A_{55}$ due to the presence of cavities occupied by SMA fibers in sleeves. Therefore, the results obtained for spacings of less than $6 \mathrm{~mm}$ should be treated as qualitative only. The problem related to degradation of the stiffnesses $A_{44}$ and $A_{55}$ may be reduced by using SMA wires of a smaller diameter with higher recovery stresses. An important conclusion from figures 6,7 and 8 is that the advantage of functionally graded SMA fibers over their uniformly distributed counterparts increases as the spacing decreases.

\section{Conclusions}

SMA fibers in sleeves may provide an effective tool for enhancement of the stability of sandwich panels subjected to the simultaneous action of mechanical and thermal loads. SMA fibers may become particularly effective at higher temperatures due to large tensile recovery stresses.

Functionally graded SMA fibers may further increase buckling loads of sandwich panels (and other structures). In the case of a uniform compression, a sinusoidal distribution of SMA fibers results in a significant increase of the buckling load. The advantage shown by functionally graded SMA fibers over their uniformly distributed counterparts increases with a decrease of spacing and an increase of temperature.

Further studies of the problem should address the following. 
V Birman

Buckling load (MN/m)

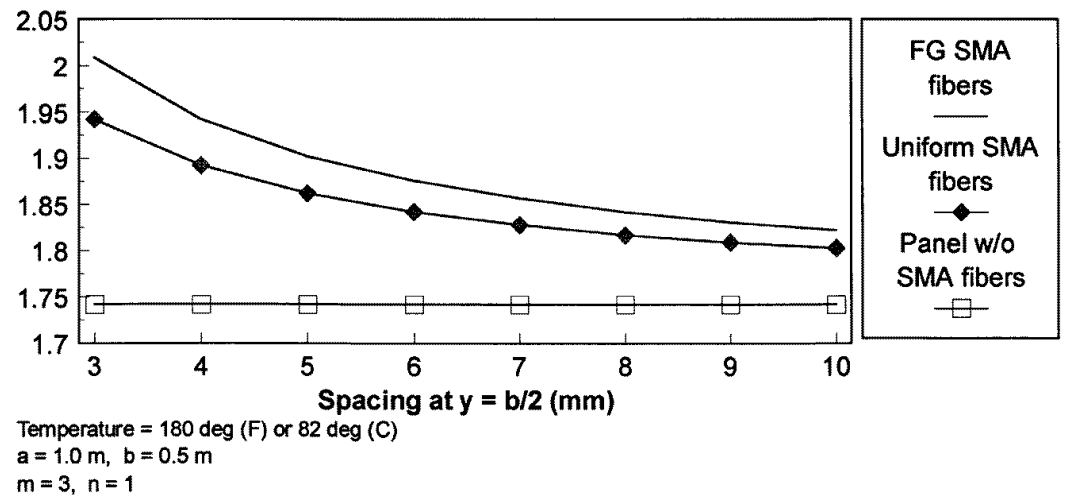

Figure 7. Effect of the spacing between SMA fibers on the buckling loads of panels with $\operatorname{Kevlar} / \mathrm{epoxy}$ facings at $T=82^{\circ} \mathrm{C}$.

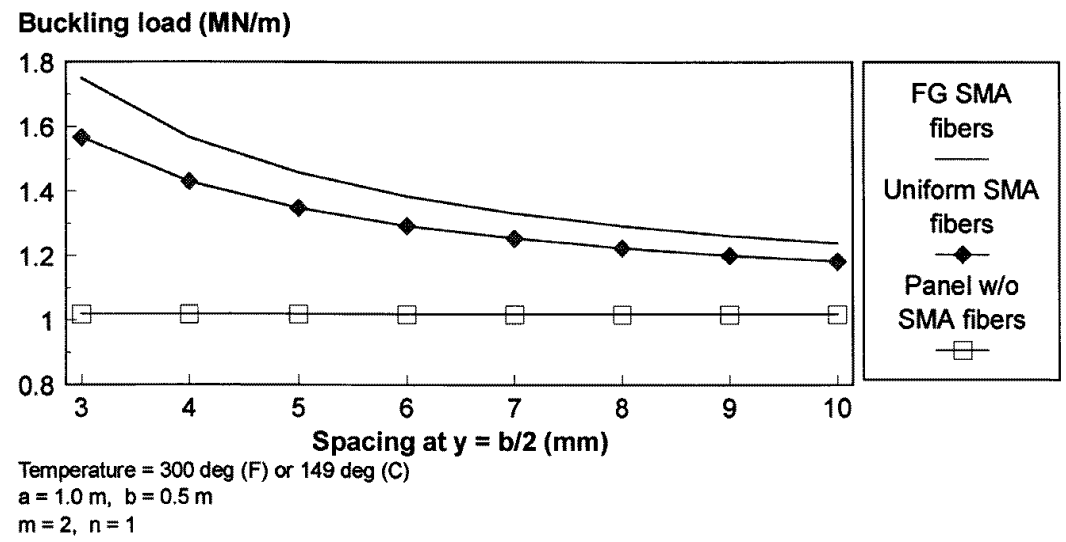

Figure 8. Effect of the spacing of SMA fibers on the buckling loads of panels with Kevlar/epoxy facings at $T=149^{\circ} \mathrm{C}$.

(1) The possibility of using SMA fibers without sleeves. The problems that have to be considered include an appropriate micromechanical theory and technological aspects.

(2) Effects of temperature on the properties of materials. In particular, the properties of polymeric materials (foam core and the matrix of composite sandwich facings) can be significantly affected even by limited fluctuations of temperature.

(3) Degradation of the core stiffnesses due to the presence of SMA fibers in sleeves. This degradation can be accounted for by using the formula for an effective modulus of shear of the core derived in appendix A.

(4) Selection of appropriate distributions of SMA fibers in various problems. The sinusoidal distribution considered in this paper is optimum for the enhancement of stability. However, this may not be the best fiber distribution in other applications. Ultimately, multi-purpose optimization should be studied as well.

The general conclusion from this work is that functionally graded smart hybrid composites show a significant potential for technological applications. These materials may offer attractive advantages in problems of buckling, static bending and dynamics.

\section{Acknowledgments}

This research was supported by the Army Research Office grant DAAH04-95-1-0142. The author would like to acknowledge the assistance of Dr Gary Anderson, Member of the Engineering and Environmental Sciences Division of the Army Research Office. Discussions with Professor Amr Baz of the Catholic University of America are also greatly appreciated.

\section{Appendix A}

The purpose of this appendix is to derive a formula for the effective shear modulus of a core weakened by the cavities occupied by the sleeves containing SMA fibers, as shown in figure 1. For this purpose, the core is subdivided into three layers (figure 9), the thickness of the middle layer being equal to the diameter of the sleeves. In addition, the actual circular cavities are replaced with equivalent cavities of a rectangular cross section (accordingly, the core is called a 'modified actual core'). The requirement that the cross sectional areas of the actual and equivalent cavities should be equal yields

$$
a=A_{s} / h_{2}
$$




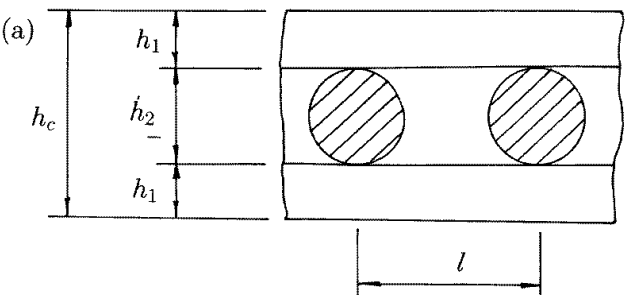

(b)

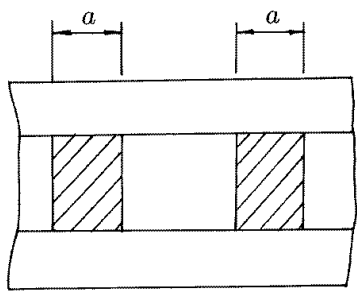

Figure 9. The core of a sandwich panel with SMA fibers in the middle plane: $(a)$ actual core; $(b)$ modified actual core.
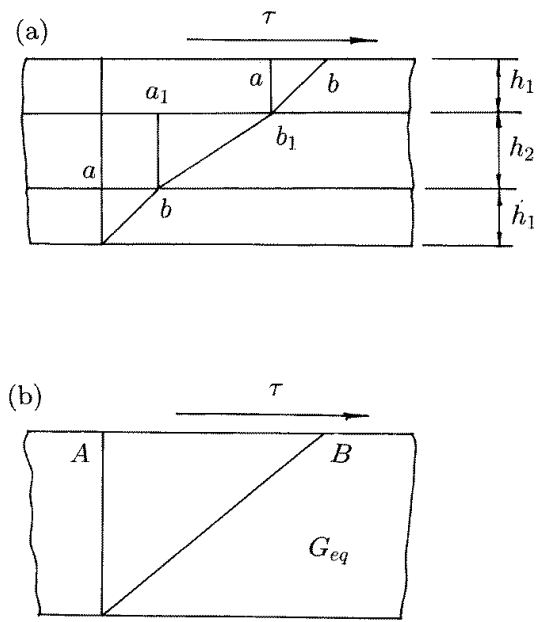

Figure 10. Shearing deformation of a modified actual core (a) and an equivalent solid core (b).

where $A_{s}$ is the cross sectional area of a sleeve containing a SMA fiber.

Consider shearing deformations of the modified actual core and those of an equivalent solid core with an equivalent shear modulus $G_{e q}$ shown in figure 10. Both cores are subjected to the action of a shearing stress $\tau$ that results in shearing deformations. For convenience, it is assumed that the bottom surfaces of the cores are clamped. Then the shearing deformations will result in a displacement of the upper surface. Obviously, this displacement should be identical for the modified actual and equivalent cores.

In the case of an equivalent core, the displacement is

$$
A B=\frac{\tau}{G_{e q}} h_{c} .
$$

In the modified actual core, the displacement of the upper surface represents the sum of the displacements $a b$ of the outer layers and the displacement $a_{1} b_{1}$ of the middle layer. The latter displacement should be calculated taking into account an increase of the shear stress due to a smaller cross sectional area. It can easily be shown that the stresses are increased by the factor

$$
d=\frac{1}{1-\bar{A}_{s} / h_{2}}
$$

where $\bar{A}_{s}=A_{s} / l$.

Accordingly, displacements $a b$ and $a_{1} b_{1}$ are

$$
\begin{aligned}
a b & =\frac{\tau}{G} h_{1} \\
a_{1} b_{1} & =d \frac{\tau}{G} h_{2} .
\end{aligned}
$$

The requirement

$$
A B=2 a b+a_{1} b_{1}
$$

yields

$$
k=\frac{G_{e q}}{G}=\frac{h_{c}}{2 h_{1}+d h_{2}} .
$$

The value of a reduction factor $k$ in the examples considered here was 0.874 , corresponding to a reduction of the shear modulus by $12.6 \%$. Note that this factor corresponds to the centerline of the sandwich, i.e. to $y=$ $b / 2$ and the immediate vicinity of this line. The factor gradually increases towards the edges $y=0$ and $y=b$ where it is equal to one.

Note that the reduction of the shear modulus of the core is relatively small. In addition, this reduction will be to some extent neutralized by a contribution from the resin sleeves and SMA fibers to the shear stiffness of the core. While this contribution is difficult to quantify, it may be used as an additional justification of the assumption that the sleeves do not significantly degrade the shear stiffness of the core.

\section{Appendix B}

The purpose of this appendix is to present proof that the optimum distribution of SMA fibers in a thin sandwich plate subjected to a combination of compressive and thermal loads is sinusoidal, i.e. $A_{f} / l=A \sin \pi y / b$. The solution is obtained for the case where the reverse phase transformation of SMA fibers is due to an external uniform temperature.

The sandwich plate considered here is thin and transverse shear stiffnesses of the core are low. Therefore, the contribution of transverse shear stiffnesses of the core to panel stability can be disregarded. SMA fibers are located at the middle plane of the panel and their effect on the bending stiffnesses is negligible. The facings are composed of a large number of angle-ply and specially orthotropic layers symmetric about the middle plane of the sandwich. In this case the equation of static equilibrium of the panel becomes

$$
\begin{gathered}
D_{11} W_{, x x x x}+2\left(D_{12}+2 D_{66}\right) w_{, x x y y}+D_{22} w_{, y y y y}-N_{x}^{r} w_{, x x} \\
+\left(N_{x}+N_{x}^{T}\right) w_{, x x}+N_{y}^{T} w_{, y y}=0 .
\end{gathered}
$$


The SMA fibers are distributed according to an arbitrary law $l(y)$ so that the recovery stress resultant can be represented by a Fourier series

$$
N_{x}^{r}=\sum_{i} N_{i} \sin \frac{i \pi y}{b}
$$

where $N_{i}$ are positive coefficients and $i$ are odd numbers (obviously, recovery forces should be symmetric about the centerline $y=b / 2$ ).

The mode shape of buckling is assumed unaffected by the recovery forces, i.e.

$$
w=W_{m} \sin \frac{m \pi x}{a} \sin \frac{\pi y}{b} .
$$

The substitution of equations (B2) and (B3) into (B1) and the Galerkin procedure result in the following expression for combination of the buckling loads:

$$
\begin{aligned}
& \left(\frac{m \pi}{a}\right)^{2}\left(N_{x}+N_{x}^{T}\right)+\left(\frac{\pi}{b}\right)^{2} N_{y}^{T} \\
& =D(m)+\frac{2}{\pi}\left(\frac{m \pi}{a}\right)^{2} \sum_{i} N_{i}\left(\frac{1}{i}-\frac{i}{i^{2}-4}\right)
\end{aligned}
$$

where $D(m)$ is a positive constant dependent on bending stiffnesses, panel dimensions and buckling mode shape.

The constraint on the volume of SMA material is expressed in the form of a limit of the recovery force:

$$
\int_{0}^{b} \sum_{i} N_{i} \sin \frac{i \pi y}{b} \mathrm{~d} y=N
$$

where $N$ is a constant. Equation (B5) yields

$$
N=\frac{2 b}{\pi} \sum_{i} \frac{N_{i}}{i} .
$$

(B6) can be substituted into the right-hand side of (B4) replacing the sum $\sum N_{i} / i$ with a positive constant. Therefore, maximum benefit to stability can be achieved by using a distribution of SMA fibers that results in a minimum of the function

$$
f=\sum_{i} N_{i} \frac{i}{i^{2}-4}
$$

proportional to the last term on the right-hand side of equation (B4).

This can be achieved if $N_{i}=0$ for all $i>1$. The corresponding value of $N_{1}$ is given by equation (B6) where $i=1$.

\section{References}

Baumgart F, Jorde J and Reiss H G 1976 Memory alloys-properties, phenomenological theory and applications NASA TM 77904 (Translation from German Technische Mittelunger Krupp, Forschungsberichte vol 34)

Baz A and Chen T 1993 Torsional stiffness of nitinol-reinforced composite drive shafts Comp. Eng. 3 1119-30
Baz A and Ro J 1992 Thermo-dynamic characteristics of nitinol-reinforced composite beams Comp. Eng. 2 $527-42$

Birman V 1995 Stability of functionally graded hybrid composite plates Comp. Eng. 5 913-21

-1997 Review of mechanics of shape memory alloy structures Appl. Mech. Rev. at press

Birman V and Adali S 1996 Vibration damping using piezoelectric stiffener-actuators with application to orthotropic plates Comp. Struct. 35 251-61

Birman V and Simonyan A 1996 Optimum distribution of actuators in smart sandwich plates subjected to bending or forced vibration Composites 27 B 657-65

Boyd J G and Lagoudas D C 1993 Thermomechanical response of shape memory alloy composites SPIE Smart Structures and Intelligent Systems vol 1917 pt II ed N W Hagood and G J Knowles pp 774-90

Brinson L C 1993 One-dimensional constitutive behavior of shape memory alloys: thermomechanical derivation with non-constant material functions and redefined martensite internal variable J. Intell. Mater. Syst. Struct. 4 229-42

Clark R L and Fuller C R 1992 Optimal placement of piezoelectric actuators and polyvinylidine fluoride error sensors in active structural acoustic control approaches $J$. Acoust. Soc. Am. 92 1521-33

Cross W B, Kariotis A H and Stimler F J 1970 Nitinol characterization study NASA CR 1433

Epps J and Chandra R 1997 Shape memory alloy actuation for active tuning of composite beams SPIE North American Conf. on Smart Structures and Materials (San Diego, California) February 26-March 31995

Jia H, Lalande, F and Rogers C A 1996 Review of constitutive modeling of shape memory alloys Proc. ASME Aerospace Division ed J G I Chang J G I et al (New York: ASME) pp 585-91

Liang C and Rogers C A 1990 One-dimensional thermomechanical constitutive relations for shape memory materials J. Intell. Mater. Syst. Struct. 1 207-34

Pates C S III, Zhong Z W and Mei C 1994 Passive control of random response of shape memory alloy fiber-reinforced composite plates Proc. 5th Int. Conf. Structural Dynamics: Recent Advances vol 1 ed N S Ferguson, H F Wolfe and C Mei The Institute of Sound and Vibration Research, University of Southampton pp 423-36

Rogers C A, Liang C and Jia J 1991 Structural modification of simply supported laminated plates using embedded shape memory alloy fibers Comput. Struct. 38 569-80

Saravanos D A, Birman V and Hopkins D A 1996 Micromechanics of composites with shape memory alloy fibers in uniform thermal fields AIAA J. 34 1905-12

Sato Y and Tanaka K 1988 Estimation of energy dissipation in alloys due to stress-induced martensitic transformation Res. Mech. 23 381-93

Tanaka K 1986 A thermomechanical sketch of shape memory effect: one dimensional tensile behavior Res. Mech. 18 251-63

White S R, Whitlock M E, Ditman J B and Hebda D A 1993 Manufacturing of adaptive graphite/epoxy structures with embedded nitinol wires Adaptive Structures and Material Systems ed G P Carman and E Garcia (New York: ASME) pp 71-9

Whitney J M 1987 Structural Analysis of Laminated Anisotropic Plates (Lancaster: Technomic)

Zhong Z W, Chen R R and Mei C 1994 Buckling and postbuckling of shape memory alloy fiber-reinforced composite plates Buckling and Postbuckling of Composite Structures ed A K Noor (New York: ASME) pp 115-32 